\title{
Non-invasive alternating current stimulation improves vision in optic neuropathy
}

\author{
Bernhard A. Sabel ${ }^{\mathrm{a}, *}$, Anton B. Fedorov ${ }^{\mathrm{a}, \mathrm{b}}$, Nicole Naue $^{\mathrm{c}}$, Antonia Borrmann ${ }^{\mathrm{a}}$, \\ Christoph Herrmann ${ }^{\mathrm{d}}$ and Carolin Gall ${ }^{\mathrm{a}}$ \\ ${ }^{a}$ Otto-von-Guericke University of Magdeburg, Medical Faculty, Institute of Medical Psychology, \\ Magdeburg, Germany \\ ${ }^{\mathrm{b}}$ EBS Technologies GmbH, Heinrich-Hertz-Straße, Kleinmachnow, Germany \\ ${ }^{\mathrm{c}}$ German Center for Neurodegenerative Diseases, Magdeburg, Germany \\ ${ }^{\mathrm{d}}$ Carl-von-Ossietzky University, Institute of Psychology, Department of Experimental Psychology, \\ Oldenburg, Germany
}

\begin{abstract}
Purpose: Partial blindness after visual system damage is considered irreversible, yet the brain has residual visual capacities and considerable plasticity potential. We now applied non-invasive alternating current stimulation (ACS) to the visual system of patients with optic nerve damage with the aim to induce recovery of visual functions.

Methods: In a prospective, double-blind, randomized, placebo-controlled clinical trial patients with several year old partial optic nerve lesions were treated with ACS $(n=12)$ or placebo-stimulation $(n=10)$. ACS was delivered transorbitally for 40 minutes on 10 days. Visual outcome measures and EEG were measured before and after treatment.

Results: ACS, but not placebo, led to significant improvement of a visual field detection deficit by $69 \%$, and also significantly improved temporal processing of visual stimuli, detection performance in static perimetry, and visual acuity. These changes were associated with alpha-band changes in the EEG power spectra. Visual improvements were stable for at least 2-months.

Conclusions: ACS can induce vision restoration many years after optic neuropathy. Though the mechanism is still unclear, EEG changes indicate increased synchronization in posterior brain regions. The present study provides Class Ib evidence that non-invasive transorbital ACS is well tolerated and improves visual function in optic neuropathy.
\end{abstract}

Keywords: Clinical trial, neuromodulation, optic nerve, vision restoration, plasticity

\section{Introduction}

Visual field defects after optic nerve or visual cortex damage show only limited recovery beyond the first weeks and months after the injury (Sabel, 1997; Zhang et al., 2006). Although partial blindness is considered permanent, some vision restoration can be achieved by intensive behavioral training (Kasten et al., 1998;

${ }^{*}$ Corresponding author: Bernhard A. Sabel, PhD, Institute of Medical Psychology, Otto-v.-Guericke University of Magdeburg, Leipziger Str. 44, 39120 Magdeburg, Germany. Tel.: +49 391672 1800; Fax: +49 391672 1803; E-mail: Bernhard.Sabel@med. ovgu.de.
Julkunen et al., 2003; Werth and Seelos, 2005; Poggel et al., 2006; Sahraie et al., 2006; Henriksson et al., 2007; Mueller et al., 2007; Gudlin et al., 2008; Marshall et al., 2008; Mueller et al., 2008; Polat, 2008; Huxlin et al., 2009; Jobke et al., 2009). This modifiability ("plasticity") of the adult visual system after injury is only possible because of considerable residual vision capacities and receptive field plasticity (Eysel et al., 1999). Residual capacities can be seen in congenitally blind patients where transcranial magnetic stimulation (TMS) induces visual phosphenes in blind retinotopic positions (Gothe et al., 2002). Furthermore, brain networks can be modified by synchronous bifocal 
TMS that increases interregional coupling in the alpha band, a modulation of interregional EEG coherence (Plewnia et al., 2008). In healthy volunteers, noninvasive transcranial direct current stimulation (tDCS) also leads to excitability changes in visual cortex and alters visual perceptual functions (Antal et al., 2003; Antal and Paulus, 2008; Chaieb et al., 2008). Following alternating current stimulation (ACS), "after-effects" in the EEG alpha-band suggest that brain stimulation affects visual processing beyond the stimulation period (Zaehle et al., 2010). Furthermore, non-invasive ACS can improve some perceptual functions in patients with optic nerve damage (Fujikado et al., 2006; Fedorov et al., 2011; Gall et al., 2010, 2011) where it increases visual fields, acuity and subjective vision.

We now report the results of our first randomized, double-blind, placebo-controlled, clinical trial which was designed to determine if repetitive transorbital ACS (rtACS) improves visual fields in optic neuropathy patients with stable visual field defects and how it affects electrophysiological functioning. Patient reported outcomes regarding subjective vision have already been reported elsewhere (Gall et al., 2011).

\section{Methods and methods}

\subsection{Patients}

Twenty-two patients with visual field loss caused by damage to the optic nerve were recruited for this prospective, randomized, double-blind, placebocontrolled clinical trial and randomized by lot to either a ACS- $(n=12)$ or placebo-group $(n=10)$.

Inclusion criteria were the presence of at least some detectable amount of residual vision (inclusion criterion), lesion age $>6$ months, i.e. beyond the period of spontaneous recovery, and a stable visual field at baseline established by repeated perimetry prior to randomization.

Exclusion criteria were heart pacemakers, any metal artefacts in head and trunk, epileptic seizures within the last three years, photosensitive epilepsy as determined by EEG, presence of an un-operated tumor, psychiatric diseases (schizophrenia etc.), unstable diabetes and diabetes causing retinopathy, high blood pressure, macular degeneration respectively maculopathy, unstable or high level of intraocular pressure $(<27 \mathrm{mmHg})$, or pathological nystagmus.

\subsection{Randomization, trial design, and classification of evidence}

This study provides Class Ib evidence that 10 days of low level non-invasive ACS is well tolerated and reduces visual dysfunction in patients with optic neuropathy. Figure 1 shows the CONSORT flow diagram. While the attending physician administering the treatment could not be blinded, the group identity was concealed from patients and diagnostic examiners. The therapist was instructed not to comment on any treatment progress. Patients were informed to which group they belonged after the final diagnostic session. All placebo patients were offered subsequent ACS. Follow-up was conducted after an 8 week treatment free interval; two patients dropped out after treatment was completed without follow-up data (Fig. 1).

\subsection{Standard protocol approvals, registrations, and patient consents}

The study was approved by an ethical standards committee on human experimentation (institutional) and written informed consent was obtained from all patients participating in the study (consent for research). All patients were treated according to the Declaration of Helsinki. This study reports on a clinical trial, which was registered at http://prsinfo. clinicaltrials.gov/, clinical trial identifier number NCT01282827.

\subsection{Treatment conditions}

The repetitive, transorbital alternating current stimulation (rtACS) was applied by four stimulation electrodes (sintered $\mathrm{Ag} / \mathrm{AgCl}$ ring electrode, Easycap, Germany) placed at or near the eyeball ("transorbital") with eyes closed. The passive electrode was positioned on the wrist of the right arm. ACS was applied with a multi-channel device (EBS Technologies, Kleinmachnow, Germany) generating weak current pulses in firing bursts of 2 to 9 pulses. The amplitude of each current pulse was below $1000 \mu \mathrm{A}$. Current intensity was individually adjusted according to how well patients perceived phosphenes. To measure individual phosphene thresholds current intensity was increased stepwise (by $10 \mu \mathrm{A}$ per second).

Stimulation frequencies were between the individual $\alpha$-range (min) and the flicker fusion frequency (max). ACS was applied daily for 10 days 


\section{CONSORT Flow Diagram}

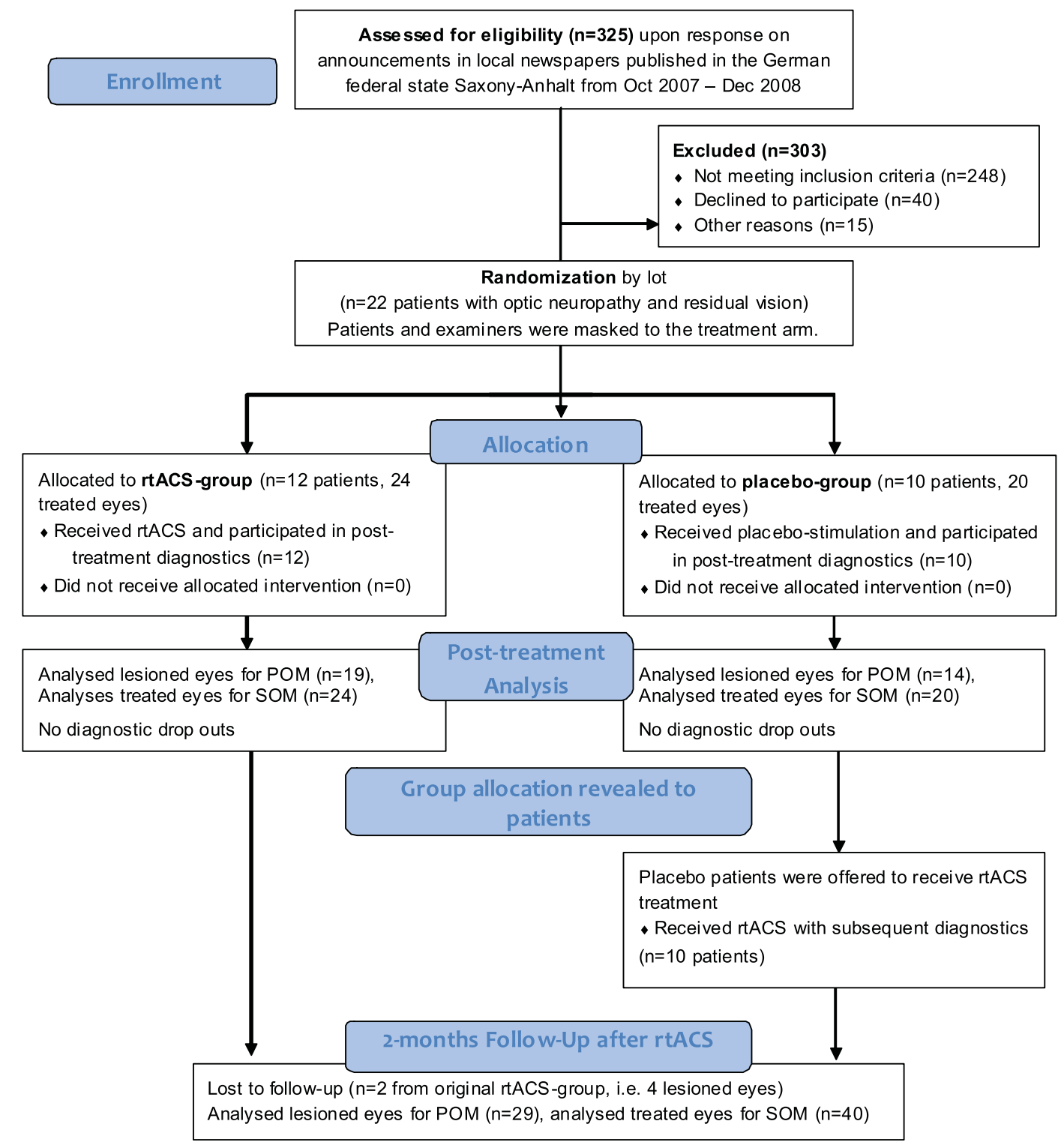

Fig. 1. Flow diagram depicting the patients' passage through the trial (adapted according to CONSORT). POM: Primary outcome measure, SOM: secondary outcome measures.

(excluding weekend). Both eyes were treated, irrespective of which eye was damaged. The length of the daily treatment session was approx. 15 min for each eye. During treatment some cutaneous sensations under the stimulation electrodes were reported by 5 patients and 4 patients had temporary sleeping difficulties. One patient had spontaneous phosphenes which were independent of stimulation during the 10-day treatment course.

To achieve the maximal possible concealment of the treatment condition, a clicking sound was presented (to generate the impression in placebo patients that 
treatment had occurred) and the same electrode montage set-up was used during ACS- and placebostimulation, but placebo patients received no current stimulation.

\subsection{Ophthalmologic examination}

Central visual fields were assessed with computerbased high-resolution perimetry (HRP) (Kasten et al., 1998). In a darkened room, patients viewed a 17 " monitor from a chin-head-rest at a distance of $42 \mathrm{~cm}$. White target stimuli were presented at random in a grid of $25 \times 19$ stimulus locations and the task was to hit the space bar. The procedure included a fixation control using isoluminant color changes of the fixation point. From these measurements visual field charts are created that display the number of hits (Fig. 2).

The ratio of correctly detected stimuli and presented stimuli, misses, false positives, fixation accuracy and reaction times were recorded. Detection accuracy (DA) change in percent above baseline within defective visual field sectors was defined as the primary outcome criterion.

Monocular visual fields were measured with a Twinfield perimeter (Oculus, Lynnwood, WA). A video camera of the perimeter was used to evaluate eye movements, pupil size and fixation ability.

During static $30^{\circ}$ perimetry 66 target stimuli (size: III $/ 4 \mathrm{~mm}^{2}$, color: white, luminance: $318 \mathrm{~cd} / \mathrm{m}^{2} / 0 \mathrm{db}$, duration: $0.2 \mathrm{sec}$ ) were presented with a fast threshold strategy on a background with constant luminance of $10 \mathrm{~cd} / \mathrm{m}^{2}$. To verify proper fixation, four target stimuli were presented inside the blind-spot, these trials were later excluded from further statistical analysis. Parameters derived from static perimetry were the foveal threshold, the mean threshold averaged across all tested positions excluding the blind spot, the number of absolute defects (misses of stimuli presented with maximum luminance), and the number of relative defects (stimulus detections at increased luminance above the physiological adequate threshold).

In kinetic perimetry the target $(0 \mathrm{~dB})$ was moved from the periphery towards fixation at a constant velocity of $2 \% \mathrm{sec}$. The visual field border was then determined for all 24 meridians randomly.

Visual acuity was measured monocularly with and without corrected refraction using a Snellen test chart at a distance of $6 \mathrm{~m}$ for distance vision and the Landoldt-ring test at a distance of $40 \mathrm{~cm}$ for near vision.

\subsection{Background EEG recordings}

EEG was recorded with a BrainAmp amplifier (Brain Products, Munich, Germany), using 32 sintered $\mathrm{Ag} / \mathrm{AgCl}$ electrodes mounted in an elastic cap (Easycap, Falk Minow Services, Munich, Germany) according to the 10-10 system, with nose-tip reference and ground electrode between Fz and $\mathrm{Cz}$. Background EEG was analyzed pre- and post-treatment with Brain Vision Analyzer, where each $60 \mathrm{sec}$ background recording was segmented in $4 \mathrm{sec}$ intervals with an overlap with the respective previous segment of $0.5 \mathrm{sec}$ while skipping intervals with artifacts. For each corrected segment Fast Fourier Transformation (FFT) analysis was performed with the following criteria: non-complex data, full spectrum, power, maximum resolution, and Hanning window with further FFT Band Export. FFT data were exported for the $7.5-13 \mathrm{~Hz}$ frequency range (alpha-rhythm) with resolution of $0.25 \mathrm{~Hz}$ for $\mathrm{O} 1$ and $\mathrm{O} 2$ electrodes. Finally, pre- and post-treatment results were analyzed to determine power spectra changes (two-sided $p$-values with alpha-adjustment). To test for a possible frequency shift after ACS, EEG profiles were created based on measured EEG amplitude with $0.5 \mathrm{~Hz}$ intervals at a frequency range of $0.5-25 \mathrm{~Hz}$ using the FFT procedure.

\subsection{Data quantification and statistical analysis}

Primary outcome measure was detection accuracy (DA) in the defective visual field sectors of HRP visual fields in percent change over baseline. Secondary outcome measures were DA in the entire visual field sector as assessed in HRP, foveal threshold and mean threshold of the total visual field, number of absolute and relative defects in static perimetry, eccentricity of visual field borders in kinetic perimetry, reaction time in HRP, and visual acuity (VA). Fixation accuracy in HRP was analyzed as an additional reliability parameter.

HRP visual field evaluations were conducted only for lesioned eyes (ACS group $N=19$, placebo group $N=14$ ) while the other tests (static and kinetic perimetry, visual acuity) were conducted with all eyes (ACS group $N=24$, placebo group $N=20$ ).

An analysis of EEG power spectra pre vs. post intervention (on the day after completing the treatment) was performed for 9 ACS and 9 placebo patients (EEG was not mandatory). 


\section{HRP visual field}

$\mathrm{Pb}$

S07 -

OD

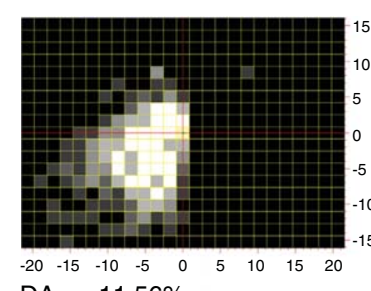

$\mathrm{DA}_{\text {pre }}=11.56 \%$

S07 - OS

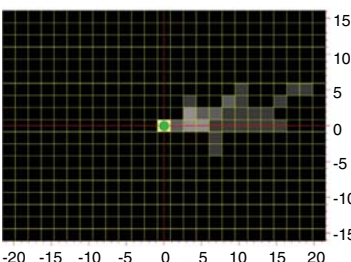

$\begin{array}{llllllllll}-20 & -15 & -10 & -5 & 0 & 5 & 10 & 15 & 20\end{array}$ $\mathrm{DA}_{\text {pre }}=1.08 \%$

S09 -

OD

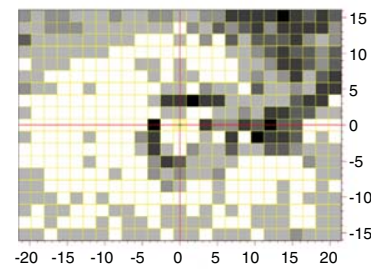

$\mathrm{DA}_{\text {pre }}=79.52 \%$

S09 - OS

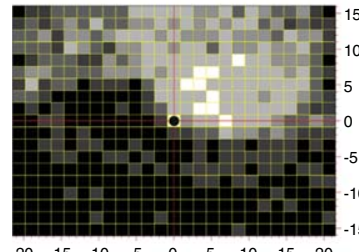

$\mathrm{DA}_{\text {pre }}=27.47 \%$

S10 -

OD

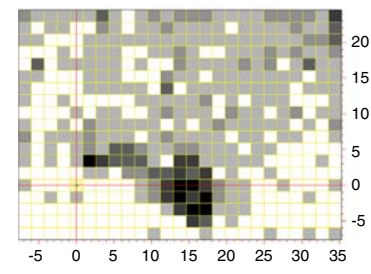

$\mathrm{DA}_{\text {pre }}=82.83 \%$

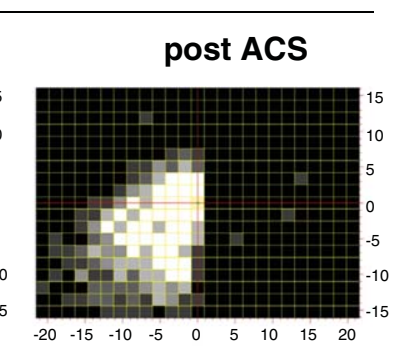

$\mathrm{DA}_{\text {post }}=15.02 \%$

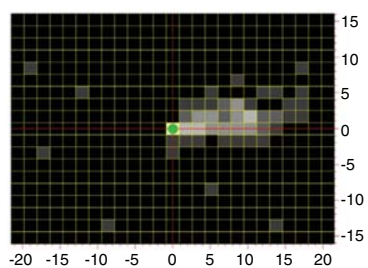

$\mathrm{DA}_{\text {post }}=2.57 \%$

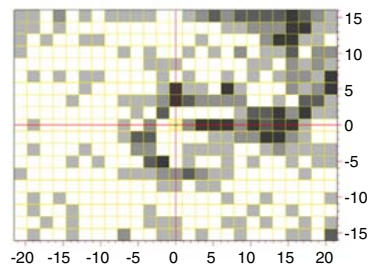

$\mathrm{DA}_{\text {post }}=86.23 \%$

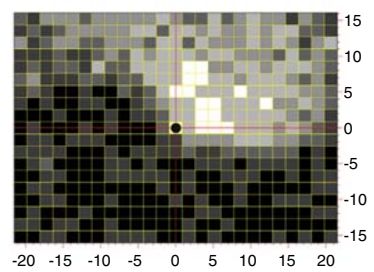

$\mathrm{DA}_{\text {post }}=29.66 \%$

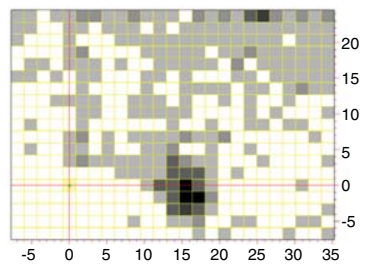

$\mathrm{DA}_{\text {post }}=88.37 \%$
Topography of visual field change (blue=increase, red=decrease of DA)

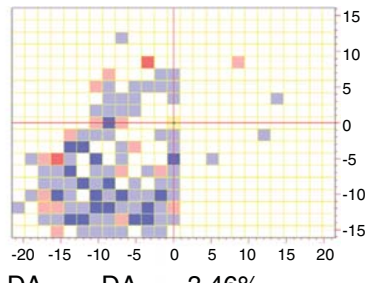

$\mathrm{DA}_{\text {post }}-\mathrm{DA}_{\text {pre }}=3.46 \%$

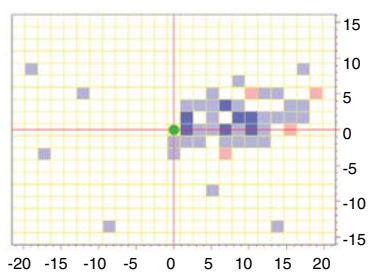

$D A_{\text {post }}-D_{\text {pre }}=1.49 \%$

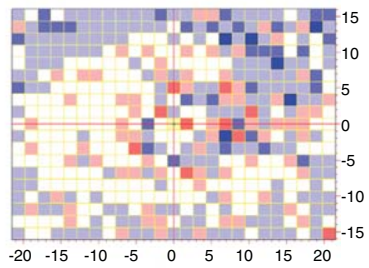

$D_{\text {post }}-D_{\text {pre }}=6.71 \%$

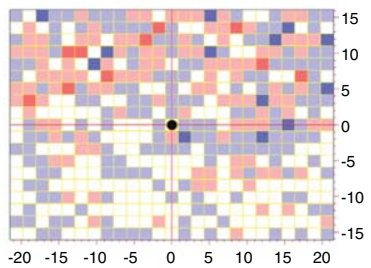

$D A_{\text {post }}-D A_{\text {pre }}=2.19 \%$

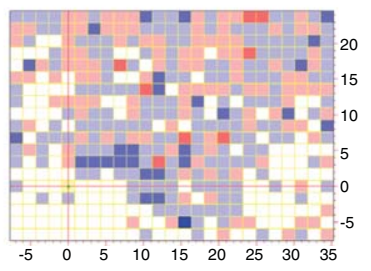

$\mathrm{DA}_{\text {post }}-\mathrm{DA}_{\text {pre }}=5.54 \%$

DA: Detection accuracy in high resolution perimetry (HRP)

Fig. 2. Visual field charts pre- vs. post stimulation. Typical topographies of changes in detection performance in the computer campimetric visual field test pre vs. post ACS. By superimposing HRP computer campimetric visual field charts of multiple measurements visual field areas were categorised as intact (perfect stimulus detection at a given location, white spots), partially damaged/relative defect (inconsistent stimulus detection, grey spots) and absolutely impaired areas (no stimulus detected, black spots). Because HRP was repeated three times, the visual field position in the chart was defined as intact when $3 / 3$ presentations were detected (white); absolutely impaired (black) were $0 / 3$, and partial (residual) vision were light (2/3) or dark gray (1/3). Based on such plots, areas of the visual field were characterized as intact, partially damaged or absolutely impaired (blind). Detection increase and decrease after ACS are shown in blue and red, respectively. 
Table 1

Comparison of patients' demographics and outcome measurements at baseline

\begin{tabular}{|c|c|c|c|}
\hline & ACS-group $(N=12)^{\dagger}$ & Placebo-group $(N=10)^{\dagger}$ & $p$ \\
\hline Age (years) & $52.3 \pm 14.3$ & $51.9 \pm 17.3$ & 0.949 \\
\hline \multirow[t]{2}{*}{ Lesion age (months) } & $68 \pm 100$ & $30 \pm 33$ & 0.265 \\
\hline & $\operatorname{ACS}(N=19 / 24)^{\ddagger}$ & Placebo $(N=14 / 20)^{\ddagger}$ & \\
\hline \multicolumn{4}{|l|}{ Computer-based HRP } \\
\hline Detection accuracy (DA) in defective visual field sectors & $24.75 \pm 22.40$ & $39.16 \pm 25.26$ & 0.094 \\
\hline Absolute detection accuracy (DA, \%) & $45.25 \pm 35.19$ & $56.95 \pm 31.03$ & 0.320 \\
\hline Fixation accuracy $(\%)$ & $80.48 \pm 29.44$ & $88.85 \pm 13.62$ & 0.285 \\
\hline Reaction time (ms) & $515 \pm 160$ & $532 \pm 126$ & 0.730 \\
\hline \multicolumn{4}{|l|}{ Automated static perimetry } \\
\hline Foveal threshold $(\mathrm{dB})$ & $17.83 \pm 9.94$ & $21.50 \pm 8.69$ & 0.199 \\
\hline Mean threshold (whole visual field, $\mathrm{dB}$ ) & $10.42 \pm 7.38$ & $13.20 \pm 6.90$ & 0.204 \\
\hline No. of absolute defects & $23.92 \pm 23.76$ & $17.15 \pm 21.28$ & 0.325 \\
\hline No. of relative defects & $13.96 \pm 11.26$ & $9.55 \pm 7.79$ & 0.134 \\
\hline \multicolumn{4}{|l|}{ Automated kinetic perimetry } \\
\hline Mean eccentricity $\left(^{\circ}\right)$ & $42.02 \pm 18.22$ & $45.86 \pm 13.31$ & 0.424 \\
\hline \multicolumn{4}{|l|}{ Visual acuity } \\
\hline Far vision & $0.46 \pm 0.48$ & $0.31 \pm 0.27$ & 0.195 \\
\hline Near vision & $0.38 \pm 0.41$ & $0.16 \pm 0.11$ & 0.023 \\
\hline
\end{tabular}

$p$-values show significance levels for between group differences (two-tailed).

${ }^{\dagger} N=$ No. of patients. ${ }^{\ddagger} N=$ No. of eyes. There were different sample sizes because computer campimetric visual field evaluations (HRP) were conducted only for lesioned eyes (ACS group: $N=19$, placebo group: $N=14$ ) while all other tests (perimetry, visual acuity) were conducted for all treated eyes (ACS group: $N=24$, placebo group: $N=20$ ).

Comparisons between groups were calculated by $t$-tests (unpaired, two-tailed) at baseline for primary and secondary outcome measures and to evaluate the changes of performance within each group (paired, one-tailed) and between them (unpaired, one-tailed). The stability of the treatment effect was evaluated also after a 2-months treatment-free interval. Results are displayed as $\mathrm{M} \pm \mathrm{SD}$.

\section{Results}

\subsection{Group comparison for outcome measures at baseline}

The ACS and the placebo group did not differ at baseline in their detection ability (DA) in the damaged sector of the visual field (primary outcome measure) and in most secondary outcome measures (Table 1). Near vision $(p=0.023)$ was slightly better in the ACS group at baseline.

\subsection{Primary outcome measure: Detection accuracy (DA) in defective visual field}

DA improvements in the defective sectors of the visual field (primary outcome criterion), were significantly larger in the ACS group (69.25 \pm $106.12 \%$, mean \pm SD) than in the placebo group $(16.93 \pm 31.22 \%)$. When the results of the ACS group were pooled with the post-ACS-treatment results of the placebo patients which received subsequent ACS treatment after a 2 months waiting-time, the improvement of both groups combined was $45.81 \pm 86.99 \%$ (Table 2; for effect size see Table 3).

\subsection{Secondary outcome measures}

As a secondary criterion DA was calculated for the entire visual field as assessed in HRP and calculated as percent change over baseline. We found a DA improvement rate of $36.79 \pm 103.62 \%$ in the ACS group (respectively all ACS-treated patients including placebo patients with consecutive ACS $(25.48 \pm 83.45 \%))$ but no change in placebo patients $(-0.84 \pm 12.99 \%)$. Between group comparisons of relative DA improvements showed a trend in this measure (Table 2).

Fixation accuracy in HRP, a quality control measure of the primary endpoint, significantly improved as well in both groups. Reaction time was also significantly reduced in ACS treated patients $(-19.63 \pm 32.96 \mathrm{~ms})$ and no such change was observed in the placebo group $(2.00 \pm 46.22 \mathrm{~ms})$. Between group comparisons of post 
Table 2

Change over baseline of primary and secondary outcome measures

\begin{tabular}{|c|c|c|c|c|}
\hline & \multicolumn{2}{|c|}{ Difference (post minus baseline) } & \multirow[t]{2}{*}{$p$} & \multirow[t]{2}{*}{$\mathrm{T}$} \\
\hline & $\overline{\operatorname{ACS}(N=19 / 24)^{\dagger}}$ & Placebo $(N=14 / 20)^{\dagger}$ & & \\
\hline \multicolumn{5}{|l|}{ Computer-based HRP } \\
\hline $\begin{array}{l}\text { Relative DA change in } \% \text { in the defective } \\
\text { visual field sectors }\end{array}$ & $69.25 \pm 106.12 * *$ & $16.93 \pm 31.22^{*}$ & 0.030 & 1.98 \\
\hline $\begin{array}{l}\text { Absolute DA change in \% (entire HRP } \\
\text { visual field) }\end{array}$ & $2.95 \pm 3.98 * *$ & $0.24 \pm 5.83$ & 0.074 & 1.50 \\
\hline $\begin{array}{l}\text { Relative DA change in \% (entire HRP } \\
\text { visual field) }\end{array}$ & $36.79 \pm 103.62$ & $-0.84 \pm 12.99$ & 0.067 & 1.56 \\
\hline Fixation accuracy $(\%)$ & $7.19 \pm 16.61 *$ & $4.40 \pm 6.96^{*}$ & 0.257 & 0.66 \\
\hline Reaction time (ms) & $-19.63 \pm 32.96^{*}$ & $2.00 \pm 46.22$ & 0.094 & -1.36 \\
\hline \multicolumn{5}{|l|}{ Automated static perimetry } \\
\hline Foveal threshold $(\mathrm{dB})$ & $0.04 \pm 2.16$ & $-0.80 \pm 2.91$ & & \\
\hline Mean threshold (whole visual field, $\mathrm{dB}$ ) & $0.54 \pm 1.11 *$ & $0.06 \pm 1.11$ & 0.080 & 1.43 \\
\hline No. of absolute defects & $-2.25 \pm 5.22 *$ & $-0.90 \pm 2.83$ & 0.141 & -1.09 \\
\hline No. of relative defects & $0.25 \pm 7.03$ & $0.60 \pm 4.46$ & 0.421 & -0.20 \\
\hline \multicolumn{5}{|l|}{ Automated kinetic perimetry } \\
\hline Mean eccentricity $\left({ }^{\circ}\right)$ & $1.19 \pm 3.57$ & $1.13 \pm 3.27$ & 0.475 & 0.06 \\
\hline \multicolumn{5}{|l|}{ Visual acuity } \\
\hline Far vision & $0.06 \pm 0.17 *$ & $-0.01 \pm 0.18$ & 0.051 & 1.67 \\
\hline Near vision & $0.07 \pm 0.18^{* * *}$ & $0.02 \pm 0.07$ & 0.019 & 2.13 \\
\hline
\end{tabular}

${ }^{\dagger} N=$ No. of eyes. For explanation of sample sizes see legend of Table 1.

*indicate significant post minus pre stimulation differences in the separate groups, ${ }^{*} p<0.05,{ }^{* *} p<0.01,{ }^{* * *} p<0.0001 ; p$-values show significance levels for between group differences of changes post intervention.

Table 3

Effect sizes for primary and secondary outcome criteria

\begin{tabular}{|c|c|c|c|c|}
\hline & Between group & \multicolumn{2}{|c|}{ Intra group (post vs. baseline) } & \multirow{2}{*}{$\begin{array}{c}\begin{array}{c}\text { Intra group (Follow-up vs. } \\
\text { baseline all ACS treated } \\
\text { patients }\end{array} \\
(N=29 / 40)^{\ddagger}\end{array}$} \\
\hline & ACS vs. Placebo & $\begin{array}{c}\text { ACS } \\
(N=19 / 24)^{\dagger}\end{array}$ & $\begin{array}{c}\text { Placebo } \\
(N=14 / 20)^{\dagger}\end{array}$ & \\
\hline \multicolumn{5}{|l|}{ HRP } \\
\hline $\begin{array}{l}\text { Relative DA change in } \% \text { in the defective } \\
\text { visual field sectors }\end{array}$ & 0.669 & 0.653 & 0.542 & 0.315 \\
\hline Absolute DA change in \% & 0.543 & 0.741 & 0.041 & 0.417 \\
\hline Relative DA change in $\%($ baseline $=100 \%)$ & 0.510 & 0.355 & 0.065 & 0.189 \\
\hline Fixation accuracy $(\%)$ & 0.219 & 0.433 & 0.632 & 0.049 \\
\hline Reaction time (ms) & 0.534 & 0.596 & 0.043 & 0.581 \\
\hline \multicolumn{5}{|l|}{ Automated static perimetry } \\
\hline Mean threshold (whole visual field, dB) & 0.432 & 0.486 & 0.054 & 0.295 \\
\hline No. of absolute defects & 0.322 & 0.431 & 0.318 & 0.394 \\
\hline No. of relative defects & 0.059 & 0.036 & 0.135 & 0.226 \\
\hline Foveal threshold $(\mathrm{dB})$ & 0.328 & 0.019 & 0.275 & 0.020 \\
\hline \multicolumn{5}{|l|}{ Automated kinetic perimetry } \\
\hline Mean eccentricity $\left(^{\circ}\right)$ & 0.018 & 0.333 & 0.346 & 0.108 \\
\hline \multicolumn{5}{|l|}{ Visual acuity } \\
\hline Far vision & 0.400 & 0.353 & 0.056 & 0.083 \\
\hline Near vision & 0.366 & 0.389 & 0.286 & 0.429 \\
\hline
\end{tabular}

Column 2 shows the effect sizes for the between group differences comparing the effects achieved by ACS vs. placebo stimulation. Columns 3 and 4 show the effect sizes for pre vs. post measurements in the ACS and placebo group. Column 5 shows the effect sizes for pre vs. follow-up in all treated patients (including former placebo patients).

${ }^{\dagger} N=$ No. of eyes at post measurement. For explanation of sample sizes see legend to Table 1.

${ }^{\ddagger} N=$ No. of eyes at follow-up. There were different sample sizes because HRP visual field evaluations were conducted only for lesioned eyes $(N=29)$ while the other tests (perimetry, visual acuity and contrast) were conducted for all treated eyes $(N=40)$. 
minus pre reaction time changes missed significance $(p=0.094)$.

ACS-patients showed a marginal increase of the mean detection threshold of $0.54 \pm 1.11 \mathrm{~dB}$ as well as a small but significant reduction of the number of absolute defects by $-2.25 \pm 5.22$ as measured by static perimetry. No significant changes were seen in placebo patients. The number of relative defects and the foveal threshold in $\mathrm{dB}$ remained unchanged in both groups and between group differences did not reach significance for static perimetry results. There was also no change of the mean eccentricity of the visual field as measured by kinetic perimetry in both groups.

Between group differences for visual acuity measures were significant for near vision and a trend towards significance was observed for far vision. While there were significant improvements in the ACS group (near vision: $0.07 \pm 0.18$; far vision: $0.06 \pm 0.17$ ), there was no significant change in the placebo group (near vision: $0.02 \pm 0.07$; far vision: $-0.01 \pm 0.18$ ) (Table 2).

\subsection{Follow-up results}

Table 4 shows the follow-up measurements comparing performance at baseline vs. after 2 months for all treated patients. For the calculation of this measure, patients of the ACS group and those placebo patients who consecutively received ACS were pooled together.
The primary outcome measure, i.e. DA change in defective visual field sectors as tested with HRP, remained elevated above baseline (36.32 $\pm 115.44 \%)$, however, slightly below significance. When the entire HRP visual field was analysed the mean absolute difference of DA was still significant at followup $(2.22 \pm 5.32 \%)$. Also the mean reaction time in HRP was still significantly faster than at baseline $(-24.76 \pm 42.58 \mathrm{~ms})$.

The mean threshold and the foveal threshold in static perimetry were significantly improved at follow-up compared to baseline and there was a trend toward a reduced number of absolute defects. However, the descriptive differences were small: reduction of number of absolute defects by $-1.30 \pm 5.74$; increase of mean threshold by $0.52 \pm 1.32$ and increase of foveal threshold by $0.90 \pm 3.05$. The number of relative defects in static perimetry and the mean eccentricity $\left(^{\circ}\right)$ in kinetic perimetry did not differ between follow-up and pre ACS measurements. Visual acuity at follow-up was still significantly improved for near but not for far vision.

\subsection{Topography of visual field improvements}

Typical topographies of DA changes before and after ACS are shown in Fig. 2. To investigate in which sector of the visual field improvements occurred, at baseline the visual field was subdivided into three regions with respect to defect depth in all responders (Fig. 3).

Table 4

Differences between follow-up and baseline for all treated patients

\begin{tabular}{|c|c|c|c|}
\hline & $\begin{array}{l}\text { Difference (Follow-up minus Baseline) } \\
\text { in ACS-treated patients }(N=29 / 40)^{\dagger}\end{array}$ & $p$ & $\mathrm{~T}$ \\
\hline \multicolumn{4}{|l|}{ HRP } \\
\hline Relative DA change in $\%$ in the defective visual field sectors & $36.32 \pm 115.44$ & 0.051 & 1.69 \\
\hline Absolute DA change in \% (entire HRP visual field) & $2.22 \pm 5.32$ & 0.016 & 2.25 \\
\hline Relative DA change in \% (entire HRP visual field) & $21.88 \pm 115.61$ & 0.159 & 1.02 \\
\hline Fixation accuracy $(\%)$ & $0.79 \pm 16.28$ & 0.398 & 0.26 \\
\hline Reaction time (ms) & $-24.76 \pm 42.58$ & 0.002 & -3.13 \\
\hline \multicolumn{4}{|l|}{ Automated static perimetry } \\
\hline Foveal threshold $(\mathrm{dB})$ & $0.90 \pm 3.05$ & 0.035 & 1.86 \\
\hline Mean threshold (whole visual field, $\mathrm{dB}$ ) & $0.52 \pm 1.32$ & 0.008 & 2.52 \\
\hline No. of absolute defects & $-1.30 \pm 5.74$ & 0.080 & -1.43 \\
\hline No. of relative defects & $-0.15 \pm 7.42$ & 0.449 & -0.13 \\
\hline \multicolumn{4}{|l|}{ Automated kinetic perimetry } \\
\hline Mean eccentricity $\left({ }^{\circ}\right)$ & $0.41 \pm 3.78$ & 0.248 & 0.69 \\
\hline \multicolumn{4}{|l|}{ Visual acuity } \\
\hline Far vision & $0.01 \pm 0.12$ & 0.293 & 0.55 \\
\hline Near vision & $0.06 \pm 0.14$ & 0.006 & 2.63 \\
\hline
\end{tabular}

$p$-values show significance levels for differences between follow-up and pre ACS measurements.

${ }^{\dagger} N=$ No. of eyes. There were different sample sizes because HRP visual field evaluations were conducted only for lesioned eyes $(N=29)$ while the remainder tests (perimetry, visual acuity and contrast) were conducted for all treated eyes $(N=40)$. 
(A) HRP (Primary outcome measure

DA change at absolute and relative defect positions)

os $(\mathrm{N}=7)$

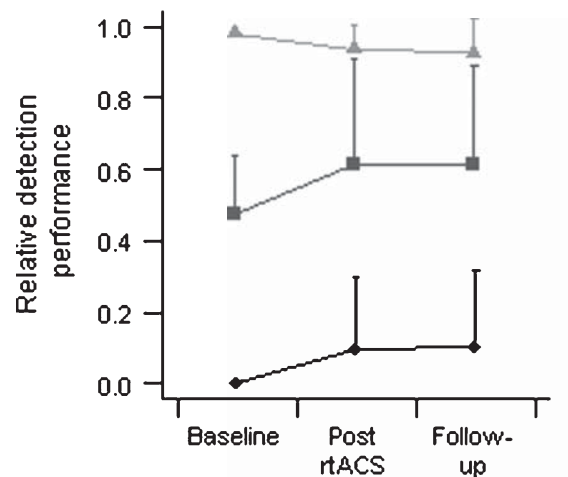

OD (N=4)

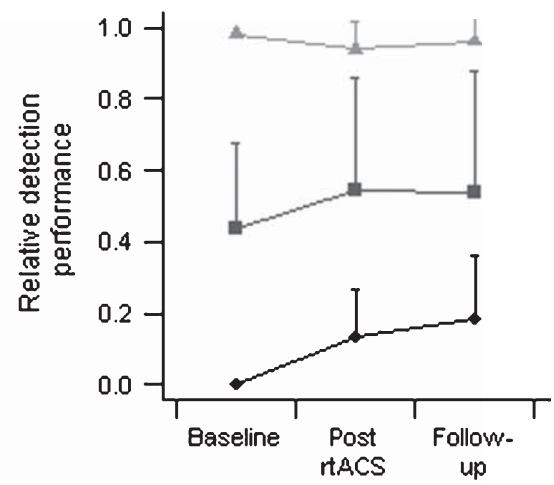

$\rightarrow$ Absolute defect positions

Relative defect positions

(inconsistent stimulus detection)

- Intact positions

(B) Standard automated perimetry (Secondary outcome measure)

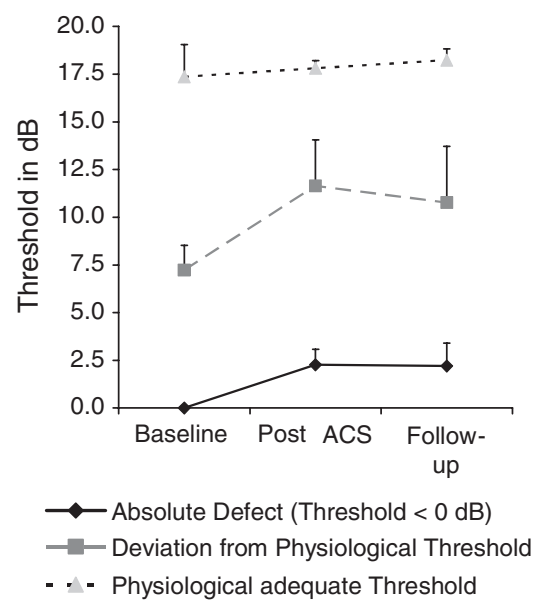

Fig. 3. Visual detection ability as a function of defect depth. Visual field results of responding patients according to the state of the visual field at baseline for (A) HRP computer perimetry and (B) standard automated perimetry. Responding patients were defined as those who showed intra-individually significant treatment results. The relative change of detection accuracy in defective visual field sectors corresponds to the overall change of absolute defect and relative defect positions (A). Results are displayed as $\mathrm{M} \pm 1 \mathrm{SE}$.

After ACS treatment DA in HRP and static perimetry improved in areas of both relative and absolute defect with stable performance at follow-up.

\subsection{EEG background analysis}

ACS-treatment increased alpha-activity at both occipital sites $(\mathrm{O} 1 / \mathrm{O} 2)$. No such changes were seen in placebo patients (Fig. 4). The alpha power spectra at $\mathrm{O} 1\left(\mu \mathrm{V}^{2} / \mathrm{Hz}\right)$ changed from $15.3 \pm 3.6$ pre to $17.1 \pm 3.5$ post ACS $(p<0.0001)$ and from $12.9 \pm 4.6$ to $15.1 \pm 3.7(p<0.0001)$ at $\mathrm{O} 2$, i.e. $11.5 \%$ and $30.4 \%$ above baseline, respectively. In the placebo group the respective values were for $01: 14.4 \pm 6.6$ pre and $14.7 \pm 5.7$ post $(p=0.629) ; \mathrm{O} 2: 13.5 \pm 7.3$ and $13.6 \pm 5.2(p=0.851)$. 
A: Between groups comparison for alpha power spectra changes
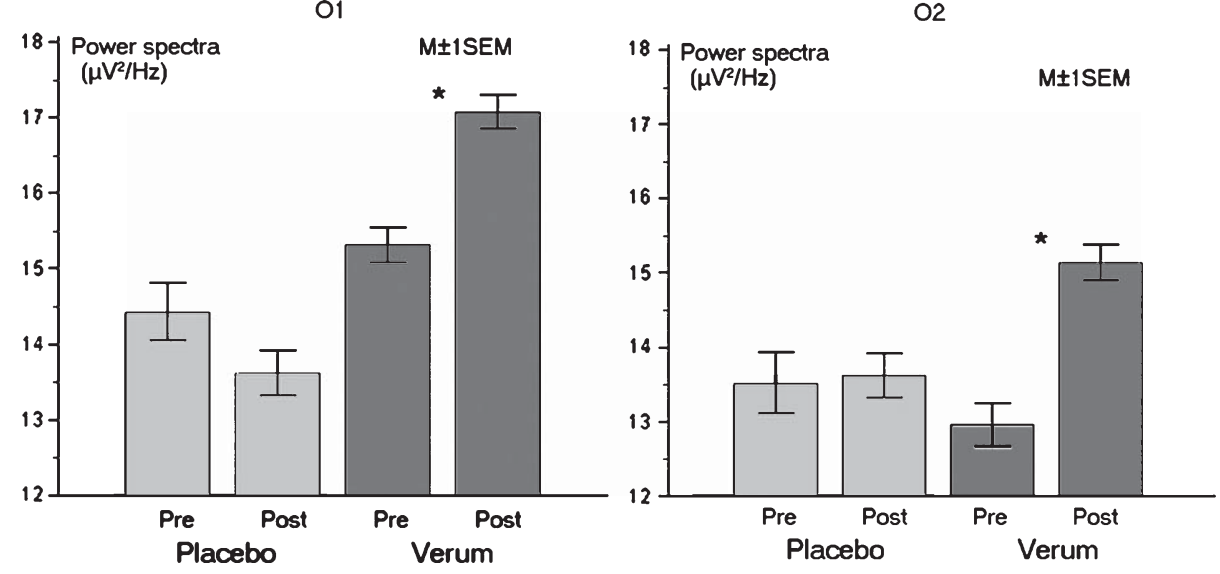

B: Spectral profiles
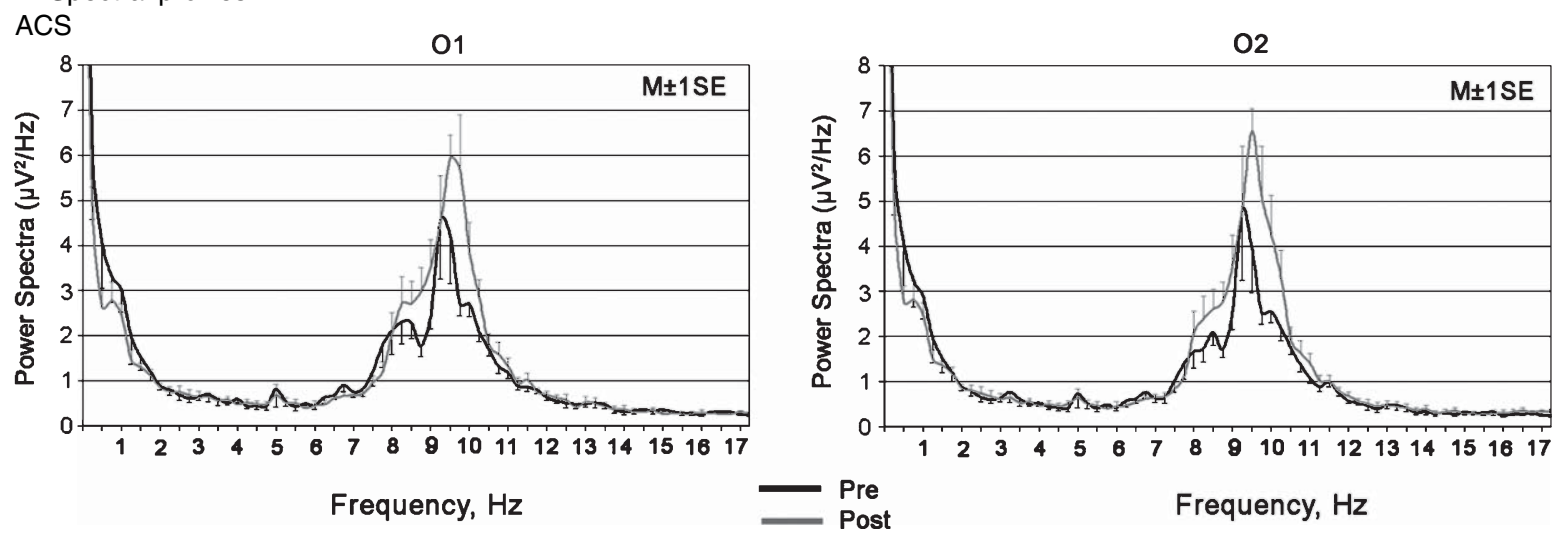

Frequency, $\mathrm{Hz}$

Frequency, $\mathrm{Hz}$
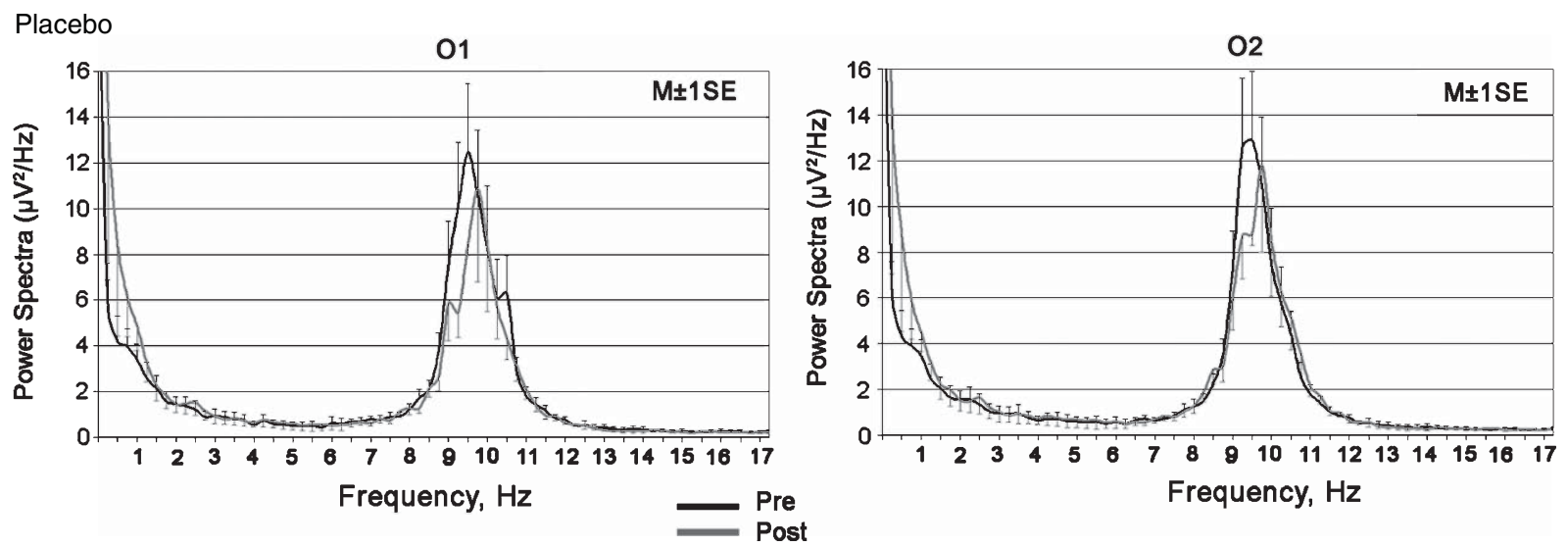

Fig. 4. EEG alpha-power spectra. Part A: Placebo vs. ACS between group analyses of alpha-rhythm power spectra changes recorded before and $24 \mathrm{hrs}$. after the last stimulation session. *Significant alpha power spectra increases were induced at O1 and O2 in the ACS group and no significant change was observed in the placebo group. Part B: EEG spectral profiles pre vs. post ACS stimulation and pre vs. post sham stimulation. Note that the power spectra range in placebo patients was approximately twice as high as in ACS patients. 
After 10 days of ACS the EEG spectral profile showed increased alpha power for the whole alpha band without a significant frequency shift at both occipital sites (paralleled by slight decrease of delta power spectra). In contrast, the spectral profile after placebo stimulation demonstrated a tendency of increased delta and decreased alpha power without involving bordering frequencies.

\section{Discussion}

Stimulation of the visual system in a non-invasive manner has been studied in Russia for several decades. Bechtereva et al. (1985) applied electrical stimulation for invasive treatment of injured optic nerves and found significant vision recovery after 3-4 weeks. Our non-invasive ACS stimulation protocol builds on this prior experience. But prior studies had neither control groups nor were they blinded (Chibisova et al., 2001; Gall et al., 2010; Fedorov et al., 2011). Our study is the first to demonstrate efficacy of ACS to reduce visual field defects after optic nerve lesions in a double-blind, placebo-controlled clinical trial. Patients showed improved stimulus detection performance in super-threshold computer-based perimetry, near-threshold perimetry, reaction time gains and near vision acuity improvements. These functional changes were stable at 2-months follow-up and were accompanied by changes in EEG power bands. Improved subjective vision of patients included in the present trial was already reported elsewhere (Gall et al., 2011).

Interestingly, visual improvements occurred mainly in areas of residual vision which is what is seen after behavioural training in hemianopic patients (Kasten et al., 1998). In these studies the extent of recovery was a function of defect depth and residual vision in the immediate surround of residual tissue (Guenther et al., 2009). Our findings are compatible with the suggestion that surviving cells in partially damaged neuronal elements play a special role in vision recovery as the extent of change was greatest in these regions (Sabel, 1997; 2011). We propose that ACS is able to somehow activate either surviving cells and/or alter the deafferented cortical regions, possibly by inducing neuronal synchronization of neuronal networks. This would explain the EEG power spectra changes we have observed in the different regions of the brain, not just in posterior regions that include visual cortex (data not shown).
But visual improvements were also observed in areas of "absolute" blindness, where they were less pronounced. In fact, some particularly striking cases of recovery from near-complete blindness exist (Gall et al., 2010). A special role of residual fibers in vision restoration inside the blind field is also suggested by the presence of "blindsight" after optic nerve damage (Wüst et al., 2002); also behavioural training is known to improve visual functions in areas of presumably "absolute" damage (Sahraie et al., 2006; Henriksson et al., 2007; Raninen et al., 2007; Jobke et al., 2009). Thus, our findings are compatible with the "residual vision activation theory" which states that surviving neuronal elements can be activated to mediate recovery of vision (Sabel et al., 2011).

Because the topography of the visual field improvements after ACS are similar to those found after training as reported previously (Kasten et al., 1998), we suggest that ACS affects physiological mechanisms which might be similar to, or identical with, long-term potentiation in perceptual learning as it is known from animal experiments. We interpret the EEG power-spectra changes as a sign of this physiological plasticity. Here, ACS, but not placebo, induced long-term changes in neurophysiological activity that may come about by enhanced synchronization of larger scale neuronal networks.

Our findings are compatible with previous vision studies in healthy volunteers where tDCS produced phosphene threshold and excitability changes in primary visual cortex and altered different visual perceptual functions (Antal et al., 2003; Antal and Paulus, 2008; Chaieb et al., 2008).

Similar effects of non-invasive current applications being able to induce excitability changes in the brain with therapeutic consequences are also reported in other functional domains (Wagner et al., 2007). In the area of motor rehabilitation after stroke, for example, both TMS and tDCS can improve function of the paretic limbs (Boggio et al., 2007; Hesse et al., 2007; Hiscock et al., 2008; Kirton et al., 2008; Yozbatiran et al., 2009). Even improvements of cognitive functions such as enhanced memory (Sparing and Mottaghy, 2008) and language functions (Martin et al., 2004) have been reported. The presumed mechanism of these effects is the induction of plasticity by increased cortical excitability perhaps through a modulation of the NMDA receptor (Paulus, 2004).

As the present study shows, non-invasive ACS can induce stable visual field improvements in patients 
with optic nerve damage and long-lasting changes of the EEG steady state, primarily in the occipital cortex. The treatment does not, however, benefit all patients equally but the response shows considerable variability between patients. Factors influencing outcome are currently under investigation in a multicenter Phase III clinical trial.

While the EEG changes indicate an impact of ACS on the physiological level, which are even more longlasting than those seen by Zaehle et al. (2010) in normal subjects, we need to further explore the mechanisms of action of these after-effects. We propose synaptic plasticity induction and neuronal network synchronization as a possible mechanism of theses clinical effects.

Patient reported outcomes revealed that many patients noted subjective improvements in every day vision (Gall et al., 2011). Significant and modest correlations were found between self-estimated visual functioning in a vision-related quality of life questionnaire and primary outcome so that the treatment effects can be considered clinically relevant.

Though more research is now required to uncover the underlying mechanisms, our findings support the theory that residual vision can be activated (Sabel et al., 2011) which was achieved here by non-invasive ACS.

\section{Acknowledgments}

We thank Sandra Heinrich, Nicole Mäter, Lisa Ernst and Eileen Poloski for their patient care and performing diagnostic measurements and technical/administrative assistance. Special thanks to Susann Sgorzaly for help with statistical analyses. Funding: The study was funded by the Otto-von-Guericke University, Magdeburg, Germany, and EBS Technologies GmbH, Kleinmachnow, Germany.

\section{References}

Antal, A., Kincses, T.Z., Nitsche, M.A. \& Paulus, W. (2003). Manipulation of phosphene thresholds by transcranial direct current stimulation in man. Exp Brain Res, 150, 375-378.

Antal, A. \& Paulus, W. (2008). Transcranial direct current stimulation and visual perception. Perception, 37, 367-374.

Bechtereva, N.P., Shandurina, A.N., Khilko, V.A., Lyskov, E.B., Matveev, Y.K., Panin, A.V. \& Nikolsky, A.V. (1985). Clinical and physiological basis for a new method underlying rehabilitation of the damaged visual nerve function by direct electric stimulation. Int J Psychophysiol, 2(4), 257-272.
Boggio, P.S., Nunes, A., Rigonatti, S.P., Nitsche, M.A., PascualLeone, A. \& Fregni, F. (2007). Repeated sessions of noninvasive brain DC stimulation is associated with motor function improvement in stroke patients. Restor Neurol Neurosci, 25(2), 123-129.

Chaieb, L., Antal, A. \& Paulus, W. (2008). Gender-specific modulation of short-term neuroplasticity in the visual cortex induced by transcranial direct current stimulation. Vis Neurosci, 25, 77-81.

Chibisova, A.N., Fedorov, A.B. \& Fedorov, N.A. (2001). Neurophysiologic features of compensatory brain process at the rehabilitation of sensoneural disturbances of visual and hearing system. Hum Physiol, 3, 14-21.

Eysel, U.T., Schweigart, G., Mittmann, T., Eyding, D., Qu, Y., Vandesande, F., Orban, G. \& Arckens, L. (1999). Reorganization in the visual cortex after retinal and cortical damage. Restor Neurol Neurosci, 15, 153-164.

Fedorov, A.B., Jobke, S., Bersnev, V., Chibisova, A., Chibisova, Y., Gall, C. \& Sabel, B.A. (2011). Restoration of vision after optic nerve lesions with non-invasive transorbital alternating current stimulation: A clinical observational study. Brain Stimul, 4, 189-201.

Fujikado, T., Morimoto, T., Matsushita, K., Shimojo, H., Okawa, Y. \& Tano, Y. (2006). Effect of transcorneal electrical stimulation in patients with nonarteritic ischemic optic neuropathy or traumatic optic neuropathy. Jpn J Ophthalmol, 50(3), 266273.

Gall, C., Fedorov, A.B., Ernst, L., Borrmann, A. \& Sabel, B.A. (2010). Repetitive transorbital alternating current stimulation (rtACS) in optic neuropathy. Neurorehabil, 27(4), 335-341.

Gall, C., Sgorzaly, S., Schmidt, S., Brandt, S., Fedorov, A. \& Sabel, B.A. (2011). Noninvasive transorbital alternating current stimulation improves subjective visual functioning and visionrelated quality of life in optic neuropathy. Brain Stimul, 4, 175188.

Gothe, J., Brandt, S.A., Irlbacher, K., Roricht, S., Sabel, B.A. \& Meyer, B.U. (2002). Changes in visual cortex excitability in blind subjects as demonstrated by transcranial magnetic stimulation. Brain, 125(Pt 3), 479-490.

Gudlin, J., Mueller, I., Thanos, S. \& Sabel, B.A. (2008). Computer based vision restoration therapy in glaucoma patients: A small open pilot study. Restor Neurol Neurosci, 26(4-5), 403-412.

Guenther, T., Mueller, I., Preuss, M., Kruse, R. \& Sabel, B.A. (2009). Treatment outcome prediction model of visual field recovery using self-organizing maps. IEEE Trans Biomed Eng, 56(3), 572-581.

Henriksson, L., Raninen, A., Näsänen, R., Hyvärinen, L. \& Vanni, S. (2007). Training-induced cortical representation of a hemianopic hemifield. J Neurol Neurosurg Psychiatry, 78(1), 74-81.

Hesse, S., Werner, C., Schonhardt, E.M., Bardeleben, A., Jenrich, W. \& Kirker, S.G. (2007). Combined transcranial direct current stimulation and robot-assisted arm training in subacute stroke patients: A pilot study. Restor Neurol Neurosci, 25(1), 9-15.

Hiscock, A., Miller, S., Rothwell, J., Tallis, R.C. \& Pomeroy, V.M. (2008). Informing dose-finding studies of repetitive transcranial magnetic stimulation to enhance motor function: A qualitative systematic review. Neurorehabil Neural Repair, 22(3), 228249. 
Huxlin, K.R., Martim, T., Kelly, K., Riley, M., Friedman, D.I., Burgin, W.S. \& Hayhoe, M. (2009). Perceptual relearning of complex visual motion after V1 damage in humans. J Neurosci, 29(13), 3981-3991.

Jobke, S., Kasten, E. \& Sabel, B.A. (2009). Vision restoration through extrastriate stimulation in patients with visual field defects: A double-blind and randomized experimental study. Neurorehabil Neural Repair, 23(3), 246-255.

Julkunen, L., Tenovuo, O., Jaaskelainen, S. \& Hamalainen, H. (2003). Rehabilitation of chronic post-stroke visual field defect with computer-assisted training: A clinical and neurophysiological study. Restor Neurol Neurosci, 21(1-2), 19-28.

Kasten, E., Wüst, S., Behrens-Baumann, W. \& Sabel, B.A. (1998). Computer-based training for the treatment of partial blindness. Nature med, 4, 1083-1087

Kirton, A., Chen, R., Friefeld, S., Gunraj, C., Pontigon, A.M. \& Deveber, G. (2008). Contralesional repetitive transcranial magnetic stimulation for chronic hemiparesis in subcortical paediatric stroke: A randomised trial. Lancet Neurol, 7(6), 507513.

Marshall, R.S., Ferrera, J.J., Barnes, A., Xian Zhang, O'Brien, K.A., Chmayssani, M., Hirsch, J., Lazar, R.M. (2008). Brain activity associated with stimulation therapy of the visual borderzone in hemianopic stroke patients. Neurorehabil Neural Repair, 22(2), 136-144.

Martin, P.I., Naeser, M.A., Theoret, H., Tormos, J.M., Nicholas, M., Kurland, J., Fregni, F., Seekins, H., Doron, K. \& Pascual-Leone, A. (2004). Transcranial magnetic stimulation as a complementary treatment for aphasia. Semin Speech Lang, 25(2), 181-191.

Mueller, I., Mast, H. \& Sabel, B.A. (2007). Recovery of visual field defects: A large clinical observational study using vision restoration therapy. Restor Neurol Neurosci, 25(5-6), 563-572.

Mueller, I., Gall, C., Kasten, E. \& Sabel, B.A. (2008). Long-term learning of visual functions in patients after brain damage. Behav Brain Res, 191(1), 32-42.

Paulus, W. (2004). Outlasting excitability shifts induced by direct current stimulation of the human brain. Suppl Clin Neurophysiol, 57, 708-714.

Plewnia, C., Rilk, A.J., Soekadar, S.R., Arfeller, C., Huber, H.S., Sauseng, P., Hummel, F. \& Gerloff, C. (2008). Enhancement of long-range EEG coherence by synchronous bifocal transcranial magnetic stimulation. Eur J Neurosci, 27(6), 1577-1583.

Poggel, D.A., Kasten, E., Mueller-Oehring, E.M., Bunzenthal, U. \& Sabel, B.A. (2006). Improving residual vision by attentional cueing in patients with brain lesions. Brain Res, 1097(1), 142148.

Polat, U. (2008). Restoration of underdeveloped cortical functions: Evidence from treatment of adult amblyopia. Restor Neurol Neurosci, 26(4-5), 413-424.

Raninen, A., Vanni, S., Hyvärinen, L. \& Näsänen, R. (2007). Temporal sensitivity in a hemianopic visual field can be improved by long-term training using flicker stimulation. J Neurol Neurosurg Psychiatry, 78(1), 66-73.

Sabel, B.A. (1997). Unrecognized potential of surviving neurons: Within-systems plasticity, recovery of function and the hypothesis of the minimal residual structure. Neuroscientist, 3, 366-370.

Sabel, B.A. Henrich-Noack, P., Fedorov, A. \& Gall, C. (2011). Vision restoration after brain damage: The "Residual Vision Activation Theory”. Prog Brain Res, 192, 199-262.

Sahraie, A., Trevethan, C.T., MacLeod, M.J., Murray, A.D., Olson, J.A. \& Weiskrantz, L. (2006). Increased sensitivity after repeated stimulation of residual spatial channels in blindsight. Proc Natl Acad Sci USA, 103(40), 14971-11476.

Sparing, R. \& Mottaghy, F.M. (2008). Noninvasive brain stimulation with transcranial magnetic or direct current stimulation (TMS/tDCS)-From insights into human memory to therapy of its dysfunction. Methods, 44(4), 329-337.

Wagner, T., Valero-Cabre, A. \& Pascual-Leone, A. (2007). Noninvasive human brain stimulation. Апnu Rev Biomed Eng, 9 527-565.

Werth, R. \& Seelos, K. (2005). Restitution of visual functions in cerebrally blind children. Neuropsychologia, 43(14), 2011-2023.

Wüst, S., Kasten, E. \& Sabel, B.A. (2002). Blindsight after optic nerve injury indicates functionality of spared fibers. $J$ Cogn Neurosci, 14, 243-253.

Yozbatiran, N., Alonso-Alonso, M., See, J., Demirtas-Tatlidede, A., Luu, D., Motiwala, R.R., Pascual-Leone, A. \& Cramer, S.C. (2009). Safety and behavioral effects of high-frequency repetitive transcranial magnetic stimulation in stroke. Stroke, 40(1), 309-312.

Zaehle, T., Rach, S. \& Herrmann, C.S. (2010) Transcranial alternating current stimulation enhances individual alpha activity in human EEG. PLoS One, 5, e13766.

Zhang, X., Kedar, S., Lynn, M.J., Newman, N.J. \& Biousse, V. (2006). Natural history of homonymous hemianopia. Neurology, 66(6), 901-905. 